\title{
Implementing a fluid volume management program to decrease intra-dialytic hypotensive events in a paediatric in-centre haemodialysis unit: a quality improvement project
}

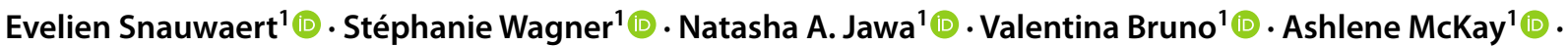

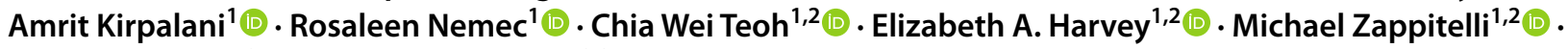 \\ Christoph Licht ${ }^{1,2}$ (1) Damien G. Noone ${ }^{1,2,3}$ (1)
}

Received: 9 May 2021 / Revised: 7 September 2021 / Accepted: 7 September 2021 / Published online: 13 October 2021

(c) The Author(s), under exclusive licence to International Pediatric Nephrology Association 2021

\begin{abstract}
Background Intra-dialytic hypotension (IDH) is the most common serious adverse event in paediatric haemodialysis (HD). Repeated IDH results in chronic multi-organ damage and increased mortality. At the Hospital for Sick Children, Toronto, retrospective data from all in-centre HD sessions revealed frequently occurring IDH events $(16.5 \pm 5.6 \%$ of HD sessions per week). Based on literature review and clinical expertise, fluid volume management was selected as a potential modifiable risk factor to decrease IDH. Root causes identified as contributing to IDH were incorporated into a Paediatric haemodialysis fluid volume management (PedHDfluid) program using the Model for Improvement methodology including rapid cycles of change. Methods Multiple measures were evaluated including (i) Outcome: IDH events per number of HD sessions per week; (ii) Process: number of changes to estimated dry weight per number of HD sessions per week; (iii) Balancing: time spent on dry weight meeting per week. Data was analysed using statistical process control charts.

We aimed to decrease IDH in our dialysis unit to $<10 \%$ of HD sessions per week over a 6 -month period by implementing a PedHDfluid program, including a multifaceted dry weight assessment protocol, multidisciplinary meetings and electronic health records "Dry Weight Evaluation flow sheet/synopsis".

Results The project resulted in a decline in IDH events from $16.5 \pm 5.6 \%$ to $8.8 \pm 3.3 \%$ of HD sessions per week. More frequent dry weight changes and increased awareness of fluid removal goals were noted.

Conclusions A multidisciplinary approach including regular assessment, guidelines and systematic discussion, with an embedded electronic health record assessment and data gathering tool may sustainably reduce IDH events.
\end{abstract}

Keywords Intra-dialytic hypotension · Haemodialysis $\cdot$ Child $\cdot$ Quality improvement

\section{Introduction}

Evelien Snauwaert and Stéphanie Wagner contributed equally to this work.

Damien G. Noone

damien.noone@sickkids.ca

1 Division of Nephrology, The Hospital for Sick Children, Toronto, ON, Canada

2 Department of Paediatrics, University of Toronto, Toronto, ON, Canada

3 Division of Paediatric Nephrology, SickKids, 555 University Avenue, Toronto, ON M5G 1X8, Canada
Intra-dialytic hypotension (IDH) is a serious and pervasive adverse event of haemodialysis procedures, defined by the Paediatric Continuous Renal Replacement Therapy working group [1] as the combination of systolic blood pressure below the 5th percentile for age and/or clinical symptoms, such as abdominal pain, nausea, vomiting, muscle cramps, restlessness, light-headedness, syncope or anxiety. IDH occurs in $9-30 \%$ of paediatric haemodialysis sessions [1, 2]. The major aetiological factor triggering IDH is intravascular volume depletion due to excessive ultrafiltration, likely in association with poor compensatory mechanisms such as abnormal cardiac function and autonomic failure [3-7]. Observational studies in adult patients on haemodialysis 
suggest that patients experiencing repeated IDH events are at risk for significant long-term cardiovascular morbidity and all-cause mortality [8-12], including myocardial infarction, reduced left ventricular ejection fraction, hospitalization, arteriovenous fistula thrombosis, intra-dialytic cerebral ischemia and decreased residual kidney function $[8,11$, 13-16]. While prospective clinical outcome data in children are lacking, minimizing the magnitude and frequency of IDH is a priority for haemodialysis providers [1].

An initial audit at our centre, The Hospital for Sick Children (SickKids, Toronto, Canada), found that IDH events occurred with an average frequency of $16.5 \pm 5.6 \%$ of haemodialysis sessions per week, confirming the existence of the problem locally. This led to the initiation of a quality improvement (QI) project in our haemodialysis unit, aiming to decrease the prevalence of IDH events to $<10 \%$ of haemodialysis sessions over the following 6 months.

A literature review conducted to identify previously reported contributing factors to IDH events found three main causes: (i) poor fluid volume management, (ii) cardiac dysfunction and (iii) autonomic dysfunction [4]. In our QI project, improving fluid volume management was selected over the other factors as it is a potentially modifiable risk factor and interventions targeting fluid volume management have been related to improved outcomes [17]. A critical component of successful fluid volume management is accurate and timely dry weight assessment, defined as the "ideal post-dialysis weight after removal of all or most of excess body fluid" [18]. Adult dialysis facilities with detailed guidelines for dry weight assessment experience lower allcause and cardiovascular mortality relative to centres without such guidelines [17]. Despite the established relationship between proper dry weight assessment and favourable outcomes, the Dialysis Outcome and Practice Patterns Study (DOPPS, 2009-2012) reported that only $25 \%$ of adult dialysis facilities in twenty countries had a protocol specifying the required frequency of dry weight assessment for their patients [17]. Moreover, assessing a patient's dry weight is fraught with difficulties, and accurate assessment of a child's dry weight can be challenging for a number of reasons. Frequent dry weight reassessment in young children is needed to keep pace with their growth. There is also limited experience in the various methods typically used in adult populations (e.g. bioelectrical impedance) that can facilitate dry weight assessment, and guidelines on dry weight assessment methodology in paediatrics are lacking [4].

Our diagnostic assessments (Cause-and-Effect diagram) identified four root causes with respect to poor fluid volume management in our dialysis cohort: (i) lack of written and well-known guidelines to determine the frequency of dry weight assessment, (ii) lack of communication between clinical team members with respect to fluid volume management, (iii) lack of team education surrounding assessment of dry weight and (iv) inefficient utilization of available resources (e.g. bioelectrical impedance) and information (e.g. orthostatic blood pressure, intra-dialytic symptoms, dietary salt and fluid intake) on dry weight assessment. We, therefore, developed a paediatric haemodialysis fluid volume management program to target these root causes. The program was embedded in the electronic health record system, Epic ${ }^{\circledR}$. We hypothesized that adequate fluid volume management with frequent and standardized dry weight assessment would result in a shift from event-driven to more systematic and preventive dry weight changes and, subsequently, a decline in IDH events (goal $<10 \%$ of HD sessions over the next 6 months).

\section{Methods}

\section{Context}

This initiative was conducted at SickKids (Toronto, Canada), a large paediatric quaternary hospital with a large in-centre HD unit, performing approximately 2000 HD sessions annually. The HD unit contains six beds and delivers four to twelve in-centre HD sessions per day, six days per week, to children aged $0-18$ years. The dialysis care providers, also our stakeholders in this project, are responsible for the fluid volume management and include six attending physicians, one or two clinical fellows (rotating bimonthly), 11 dialysis nurses and one dietician. Importantly, the COVID-19 pandemic occurred shortly after initiation of this QI project, which resulted in unanticipated effects during March and May 2020 (see discussion section for details). The dialysate temperature was standard $36.5{ }^{\circ} \mathrm{C}$ and did not change throughout the project.

\section{Intra-dialytic hypotension prevalence and dry weight assessment prior to intervention}

We retrospectively reviewed charts of all dialysis sessions ( $n=553,13$ patients) for 15 weeks prior to initiation of any intervention. IDH events, defined by intra-dialytic systolic blood pressure $<5$ th percentile for age and/or symptoms triggering a fluid bolus administration or cessation of ultrafiltration, were frequent, with events occurring during $16.5 \pm$ $5.6 \%$ of sessions per week ( 91 sessions total). Fluid boluses were administered during $3.3 \pm 4.4 \%$ of sessions per week (18 sessions total), ultrafiltration was stopped during $12.5 \pm$ $6.8 \%$ of sessions per week (69 sessions total), and hypotension was documented in $6.0 \pm 3.6 \%$ per week (33 sessions total). During this interval, dry weight was changed with a weekly average frequency of $3.9 \pm 3.7$ changes per 100 sessions. 


\section{Development of a paediatric haemodialysis fluid volume management program}

This study employed the Model for Improvement methodology. As detailed in Supplementary Table 2S, six Plan-DoStudy-Act (PDSA) cycles were conducted over a four-month period from February 2020 to June 2020 to develop and iteratively refine a multifaceted Paediatric haemodialysis fluid volume management (PedHDfluid) program. As depicted in Supplementary Table 1S, the final program included three phases: PHASE I: a multidisciplinary dry weight meeting with a newly formulated standardized dry weight assessment; PHASE II: the implementation of a dry weight flowsheet embedded in the Epic ${ }^{\circledR}$ electronic health record program; and PHASE III: the development of an introduction presentation for new fellows and dialysis nurses.

\section{PHASE I: multidisciplinary dry weight meetings with standardized dry weight assessment}

During Phase I ( 9 weeks), we focused on (multidisciplinary) communication, education and efficient use of available resources. To enhance multidisciplinary team discussions and communication and stimulate fellows' education on dry weight assessment, a multidisciplinary dry weight meeting (PDSA \#1) was initiated. The meetings were attended by dialysis attending physician(s), clinical trainee(s), a dietitian and nurse(s) and were scheduled weekly (30-45 min). During these meetings, three to four patients were discussed and time for teaching for clinical trainees, and dialysis personnel was incorporated. During Phase I, the COVID-19 pandemic started and adaptations on the concept were needed: dry weight meetings were continued but were pivoted to a virtual platform (Microsoft Teams®).

To tackle the lack of confidence of paediatric nephrology trainees on dry weight assessment and the absence of a guideline on dry weight assessment in the unit, a standardized dry weight assessment (PDSA \#2) was developed during the first three dry weight meetings. The newly standardized dry weight assessment was developed using available resources in the unit, available evidence from the literature on paediatric dry weight assessment and stakeholder consensus. Consensus was obtained on systematic IDH events tracking, required parameters (clinical measures, lab results and treatment regimen) for dry weight assessment and frequency of dry weight assessment based on patient characteristics. An overview of all aspects of the standardized dry weight assessment, including frequency of dry weight assessment, is summarized in Supplementary Table 3S. After implementation of Phase I, statistical process control (SPC) charts were reassessed after 15 consecutive weeks.

At the end of Phase I, stakeholders reported concerns that (i) relevant variables for dry weight assessment were not easily accessible in electronic patient charts, (ii) decisions made in dry weight meetings were not always translated in practice, (iii) decisions in dry weight rounds could not be clearly tracked and (iv) there was significant preparation time needed to collate the required parameters for each patient (estimated 30-45 min per patient) before dry weight round.

\section{PHASE II: automatically generated dry weight flow sheet}

In Phase II (10 weeks), we focused on improving communication and efficiency in response to concerns raised in the first phase. Therefore, we leveraged our institution's electronic health record (Epic $\left.{ }^{\circledR}\right)$ to create (i) a "Dry Weight Evaluation" flowsheet to document decisions made during the assessment of a patient's fluid volume status (PDSA \#3, see Fig. 1) and (ii) a "Dry Weight Assessment" Synopsis that automatically displayed relevant patient data on one screen to facilitate an efficient assessment of a patient's fluid volume status and to track dry weight assessment decisions (taken from "Dry Weight Evaluation" flowsheet) longitudinally over time (PDSA \#3, see Figs. 1 and 2). Within Epic $®$, flowsheets are customizable documentation tools that collect a variety of data discretely and Synopsis is a review tool that displays different types of patient data (i.e. vital signs, labs, medications and discrete data collected in specific flowsheets) that are automatically populated from the patient chart and summarized in one place. Synopsis also facilitates longitudinal tracking and allows users to review trends (graphically) for specific parameters over time (see Fig. 2). The "Dry Weight Evaluation" flowsheet and "Dry Weight Assessment" Synopsis were created by the institution's Epic $®$ development team in close collaboration with subject matter experts from paediatric nephrology (one of whom is an Epic ${ }^{\circledR}$ certified Physician Builder) and the haemodialysis unit, and reviewed by all stakeholders.

Second, responsibilities of all team members were defined to ensure translation of decisions into practice (PDSA \#4). Transparent scheduling and summaries of the meetings were organized to ensure good communication between all team members.

\section{PHASE III: an introductory presentation for new fellows and dialysis nurses}

During Phase III ( 2 weeks), efficient utilization of resources and education was further refined. To enhance the efficiency of the "Dry Weight Evaluation" flow sheet and Dry Weight Assessment" Synopsis in practice, we extended its use beyond the scheduled dry weight meetings and incorporated assessments after every (minor) event or fluid-related symptoms (PDSA \#5). 


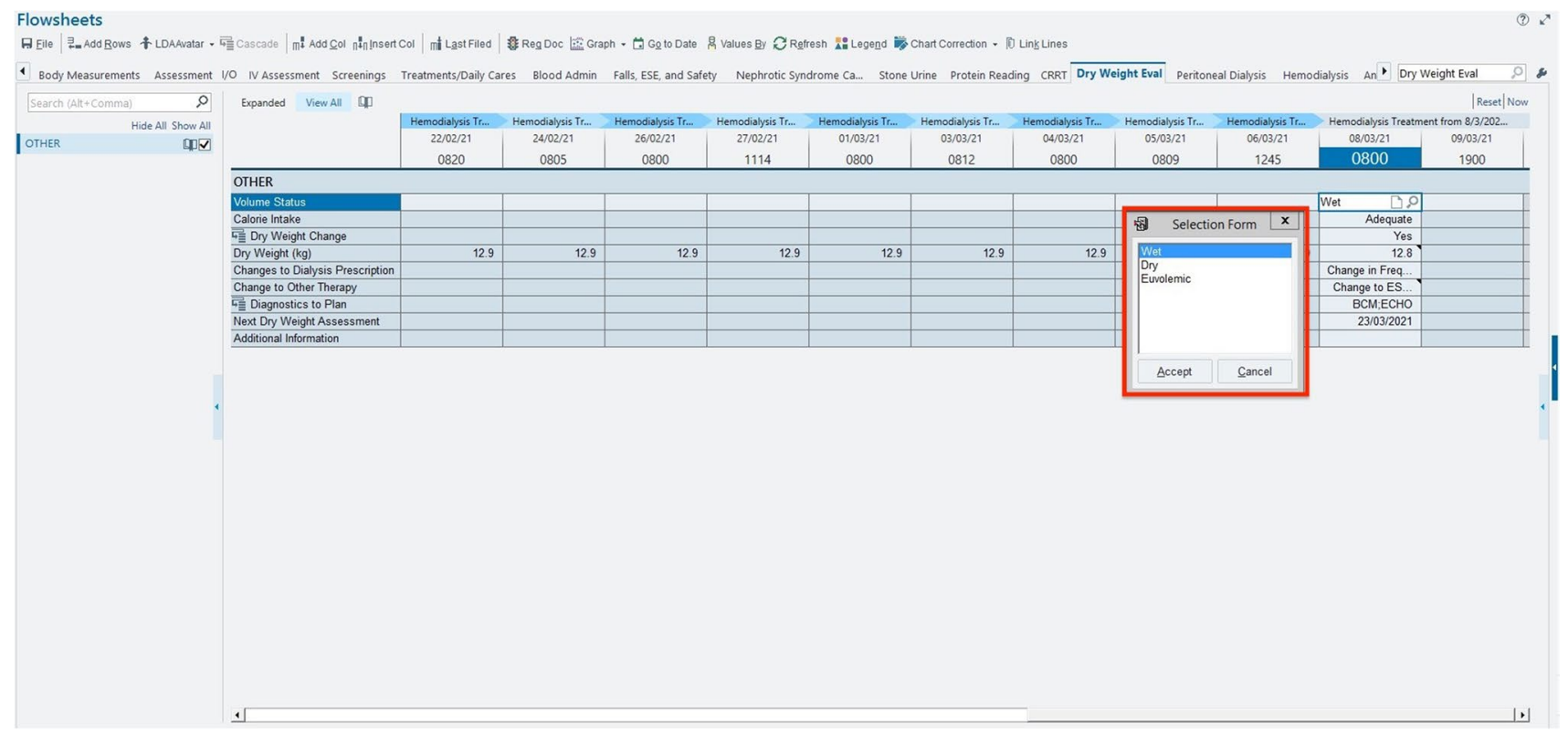

Fig. 1 Epic ${ }^{\circledR}$ screenshots. Dry Weight Evaluation flowsheet to document decisions and changes from fluid volume status assessment. Fields are populated by free text or by selecting from specific options (see example of pop-up window highlighted by red borders)

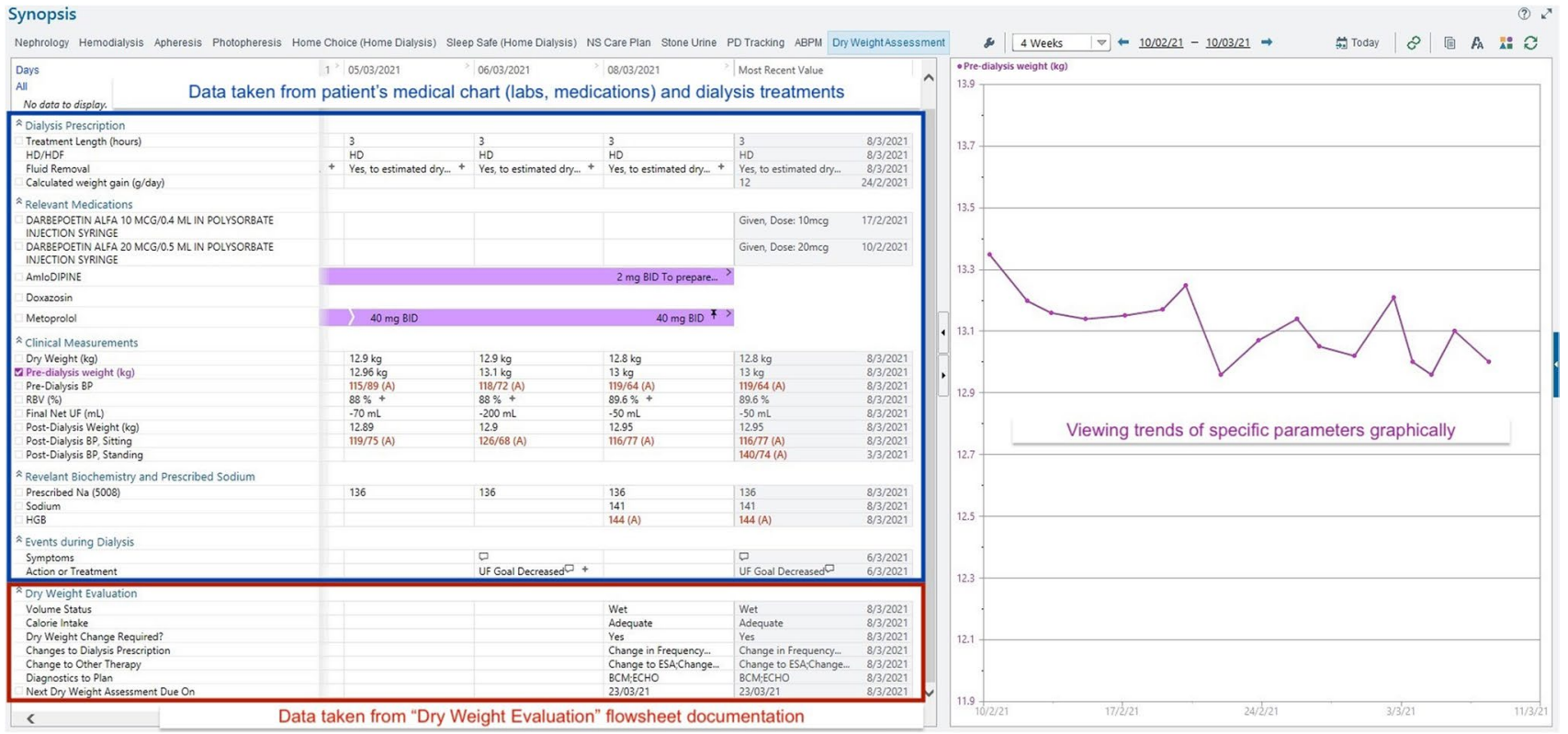

Fig. 2 Epic ${ }^{\circledR}$ screenshots. Dry Weight Assessment Synopsis that is auto-populated with data taken from various parts of the patient medical records (section highlighted in blue) and from Dry Weight Evaluation flowsheet (section highlighted in red). This Synopsis activity

During Phase II, concerns were expressed on the sustainability of the project as the dialysis unit highly depended on trainees who rotated through different services and have different background experience in facilitates an efficient fluid volume status assessment by summarizing relevant data on one screen and allows for longitudinal tracking of decisions made. It also facilitates longitudinal trending of specific parameters graphically

paediatric haemodialysis. Therefore, in PDSA \#6, an introductory presentation was developed for orienting junior fellows and dialysis nurses to the protocol, resources and expectations with respect to paediatric fluid volume management. 


\section{Family of measures}

\section{Outcome}

The number of IDH events per number of HD sessions (expressed as a percentage) per week was selected as the primary outcome measure of this project. IDH events were defined according to recent consensus guidelines [1] by intra-dialytic systolic blood pressure $<5$ th percentile for age and/or symptoms (e.g. abdominal pain, nausea, vomiting, muscle cramps, restlessness, light-headedness, syncope or anxiety) triggering a fluid bolus administration or cessation of ultrafiltration. Secondary outcome measures were fluid bolus administration and the need to stop ultrafiltration in response to symptoms likely attributable to hypotension.

\section{Process}

As the overall intent of the newly developed multifaceted interventional PedHDfluid program was to create a shift from eventdriven to more systematic and preventive dry weight changes, we expected an increase in dry weight changes after implementation of the PedHDfluid program. Therefore, the number of dry weight changes per number of HD sessions (expressed as a percentage) per week was used as a process measure in the project.

\section{Balancing}

Time spent on dry weight meetings per week and its preparation time were used as balancing measures. All measures were collected on a weekly basis (inclusive of all dialysis sessions of that week) during a 15-week period before (retrospective) and 30-week period after (prospective) initiation of the PedHDfluid protocol. Moreover, pre-dialysis systolic and diastolic blood pressures (expressed in percentiles according to age and height [19]) and antihypertensive agent use were tracked during the baseline and the post-intervention period to monitor the incidence of hypertension and fluid overload.

\section{Analysis}

Statistical process control charts were used to analyse our outcome, process and balancing measures. Established rules for differentiating special vs. common cause variation were employed [20]. Family of measures at baseline and after implementation of the PedHDfluid program were compared using non-parametric Mann-Whitney $U$ test. A $P$ value $<0.05$ was considered statistically significant. Bonferroni correction was applied to account for multiple testing. All statistical analyses were performed using $\mathrm{R}$ version 3.1.1 (R Project for Statistical Computing, Vienna, Austria). Standards for Quality Improvement Reporting Excellence Guidelines (v2.0 SQUIRE) were applied [21, 22].

\section{Ethical aspects}

This study was reviewed and approved through The Hospital for Sick Children's Quality Improvement Review Process (Toronto, Canada). The requirement for consent was waived.

\section{Results}

Over the course of the project ( 30 weeks after initiation of Phase I, between February and September 2020), 1094 dialysis sessions (total of $3953 \mathrm{~h}$ of dialysis) were performed. As summarized in Table 1, 20 children (mean age $10.8[8.3 ; 15.0] ; 1.5 \% \leq 3$ years and $40 \%$ male) were treated with haemodialysis in the HD unit. Glomerulopathy (35\%) was the most frequent underlying kidney disease. Vascular access was obtained with central venous line in all patients. At baseline, $40 \%$ of patients were treated with 1 or more antihypertensive agents and left ventricle hypertrophy was present in $5 / 20(25 \%)$ of patients. There was no missing data throughout this data collection period.

\section{Outcome}

We found a significant decline in IDH events from $16.5 \pm 5.6 \%$ of HD sessions per week at baseline, to $8.8 \pm 3.3 \%$ of HD sessions per week after implementation of the PedHDfluid protocol $(p<0.05)$ (Table 2). As visualized in the control chart (Fig. 3), this effect was sustained for approximately 3 months (July to September 2020). Similarly, the implementation of the PedHDfluid program resulted in changes in our secondary outcome measures: a reduction in hypotension rates from $6.0 \pm 3.6 \%$ of HD sessions per week at baseline to $1.9 \pm 2.0 \%$ of HD sessions per week postintervention $(p<0.05)$ and a significant decline in episodes of ultrafiltration cessation $(13.3 \pm 6.6 \%$ pre- and $7.4 \pm 2.9 \%$ post-intervention, $p<0.05$ ) was noted. No change in fluid bolus administration was seen throughout the project. The average treatment time per session and the average dialysis frequency remained similar throughout the project (Table 2).

\section{Process}

As expected, the implementation of the PedHDfluid program resulted in more dry weight changes per number of HD sessions per week (process measure) relative to baseline $(9.8 \pm 3.8 \%$ post- versus $3.9 \pm 3.7 \%$ pre-intervention, $p<0.05$ ) (Table 2 and Fig. 4).

\section{Balancing}

As displayed in table 2, the time spent during dry weight meetings was $30 \pm 12$ min per meeting (discussing 3-4 
Table 1 Patient demographics

\begin{tabular}{ll}
\hline Number & 20 \\
Male & $8(40 \%)$ \\
Age at baseline (years) & $10.8[8.3 ; 15.0]$ \\
Underlying kidney disease & \\
CAKUT & $6(30 \%)$ \\
Cystic disease, ciliopathy & $4(20 \%)$ \\
Glomerulonephritis, glomerular disease & $7(35 \%)$ \\
Others & $3(15 \%)$ \\
Dialysis vintage at baseline (months) & $21[8 ; 26]$ \\
Vascular access & \\
Central venous line & $20(100 \%)$ \\
Arteriovenous fistula & $0(0 \%)$ \\
Pathology on echocardiogram at baseline & \\
Signs of left ventricle hypertrophy present & $5(25 \%)$ \\
Residual kidney function & \\
$<200$ mL/24u & $5(25 \%)$ \\
$200-500$ mL/24u & $5(25 \%)$ \\
$>500$ mL/24u & $10(50 \%)$ \\
Number of antihypertensive therapies at baseline & \\
0 & $12(60 \%)$ \\
$1-2$ & $6(30 \%)$ \\
Number of patients treated with midodrine throughout the project & $2(10 \%)$ \\
Number of dialysis sessions performed during follow-up period & $1(5 \%)$ \\
Total number of dialysis hours during follow-up period & 30 \\
\hline
\end{tabular}

Data are median [25th; 75 th percentile] or mean \pm SD, as appropriate, and categorical variables are expressed as frequencies and percentages

patients), which was considered acceptable by all stakeholders. The preparation time for the dry weight meetings in Phase I were approximately $30-45$ min per patient, which was perceived to be too long. After implementation of Phase II, the preparation time dropped to 5-10 min per patient. The proportion of patients with pre-dialysis systolic and diastolic hypertension remained stable throughout the project. Moreover, a drop of antihypertensive agents use was noted from $22.1 \%$ to $14.8 \%$ of all HD sessions per week.

\section{Discussion}

This QI project evaluated the impact of a paediatric-specific fluid volume management (PedHDfluid) program on intra-dialytic hypotension in a large paediatric quaternary in-centre HD unit. Historically, IDH events were symptomatic and contributed to poor tolerance of HD sessions in our centre, and excessive fluid removal has previously been shown to result in increased hospitalizations, patient discomfort and substantial cost [10, 23-26]. Our PedHDfluid program resulted in a critical decline in IDH events, hypotensive episodes and ultrafiltration cessation; and a shift from sporadic, event-driven dry weight changes to systematic and preventative changes. Our results emphasize the importance of consistent clinical assessment of target weight and fluid balance in the HD unit, which is in line with the findings of the recent observational study by Dasgupta et al. [17]. This group found that while the routine use of online volume indicators or sodium modelling/profiling were associated with increased hospitalization and mortality, having a protocol that specifies frequency and method of dry weight assessment resulted in a lower risk for all-cause and cardiovascular mortality [17].

This study showed a significant decline in our primary outcome measure, IDH events, from $16.5 \pm 5.6 \%$ to $8.8 \pm 3.3 \%$ of HD sessions per week after implementation of the PedHDfluid program, thus, achieving our predefined target of $<10 \%$ of HD sessions per week. Furthermore, we were able to show that the impact of our intervention on reducing IDH events was sustainable over a period of three months following implementation. Similarly, the PedHDfluid program significantly reduced hypotension rates and ultrafiltration cessation: hypotension rates dropped from $6.0 \pm 3.6 \%$ to $1.9 \pm 2.0 \%$ post-intervention, and ultrafiltration cessation rates were only $7.4 \pm 2.9 \%$ of HD sessions 
Table 2 Overview outcome, balancing and process measures at baseline and after implementation (after Phase III) of Paediatric haemodialysis fluid volume management project

\begin{tabular}{|c|c|c|}
\hline & Baseline & $\begin{array}{l}\text { Post-interven- } \\
\text { tion (after Phase } \\
\text { III) }\end{array}$ \\
\hline \multicolumn{3}{|l|}{ General information } \\
\hline Time period & 15 weeks & 11 weeks \\
\hline Total number of dialysis sessions performed & 553 & 419 \\
\hline Average number of dialysis sessions per week & $36.9 \pm 2.2$ & $38.1 \pm 3.3$ \\
\hline Total dialysis hours (hours) & 1948 & 1452 \\
\hline Average treatment time per session (hours) & $3.5 \pm 0.3$ & $3.5 \pm 0.2$ \\
\hline Average dialysis frequency & $3.5 \pm 0.1$ & $3.4 \pm 0.2$ \\
\hline \multicolumn{3}{|l|}{ Outcome measure } \\
\hline IDH events ( $\%$ of all HD sessions per week) & $16.5 \pm 5.6$ & $8.8 \pm 3.3 *$ \\
\hline Fluid bolus ( $\%$ of all HD sessions per week) & $2.6 \pm 2.4$ & $4.3 \pm 3.2$ \\
\hline UF stop (\% of all HD sessions per week) & $13.3 \pm 6.6$ & $7.4 \pm 2.9 *$ \\
\hline Hypotension (\% of all HD sessions per week) & $6.0 \pm 3.6$ & $1.9 \pm 2.0^{*}$ \\
\hline \multicolumn{3}{|l|}{ Process measure } \\
\hline DW changes ( $\%$ of all HD sessions per week) & $3.9 \pm 3.7$ & $9.8 \pm 3.8 *$ \\
\hline \multicolumn{3}{|l|}{ Balancing measure } \\
\hline Time DW meeting (minutes per week, discussing 3-4 patients) & 0 & $29.8 \pm 11.9^{*}$ \\
\hline $\begin{array}{l}\text { Number of patients with systolic blood pressure }>95 \text { th percentile (\% of } \\
\text { all HD sessions per week) }\end{array}$ & $15.3 \pm 2.2$ & $19.6 \pm 3.3$ \\
\hline $\begin{array}{l}\text { Number of patients with diastolic blood pressure }>95 \text { th percentile }(\% \\
\text { of all HD sessions per week) }\end{array}$ & $11.7 \pm 0.5$ & $14.4 \pm 4.4$ \\
\hline Number of patients on antihypertensive agents & 8 & 6 \\
\hline Number of antihypertensive agents (\% of all HD sessions per week) & $22.1 \pm 1.4$ & $14.8 \pm 0.9^{*}$ \\
\hline
\end{tabular}

Data are mean \pm standard deviation

${ }^{*} p<0.05$, comparing measures at baseline versus after intervention

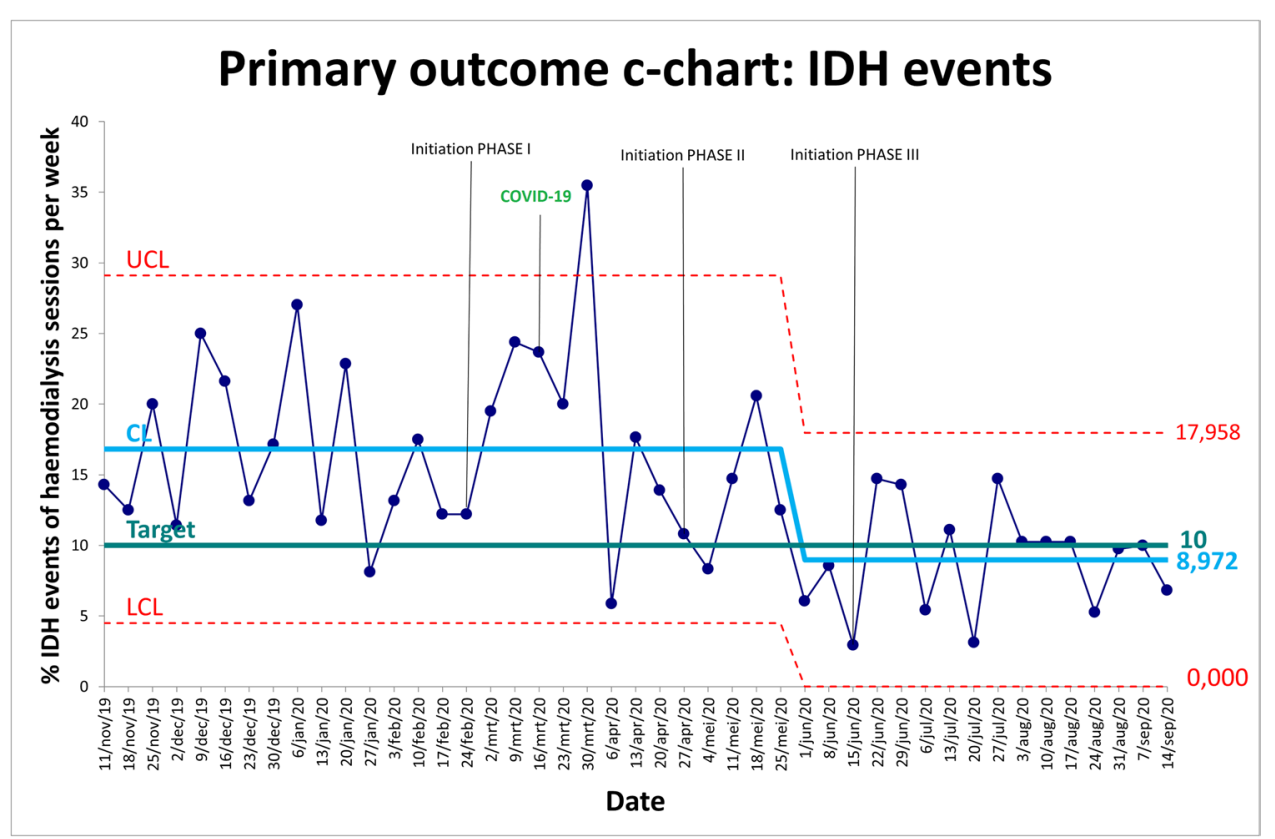

Fig. 3 Statistical control chart (c-chart) of primary outcome measure: IDH events. Red-dotted lines represent, respectively, upper control limit (UCL, $+3 \sigma$ to mean) and lower control limit (LCL, $-3 \sigma$ from mean). Light blue line represents control line (CL, mean). Green line represents target set in the aim statement: Decrease IDH $<10 \%$ of haemodialysis sessions per week. PHASE I: Multidisciplinary dry weight meetings with standardized dry weight assessment. PHASE II: Automatically generated dry weight flow sheet. PHASE III: An introductory presentation for new fellows and dialysis nurses. IDH: intra-dialytic hypotension. COVID-19: presents time point of changes in the dialysis unit due to the COVID-19 pandemic, identified as a contextual factor that may have interfered with our project 


\section{Process measure c-chart: dry weight changes}

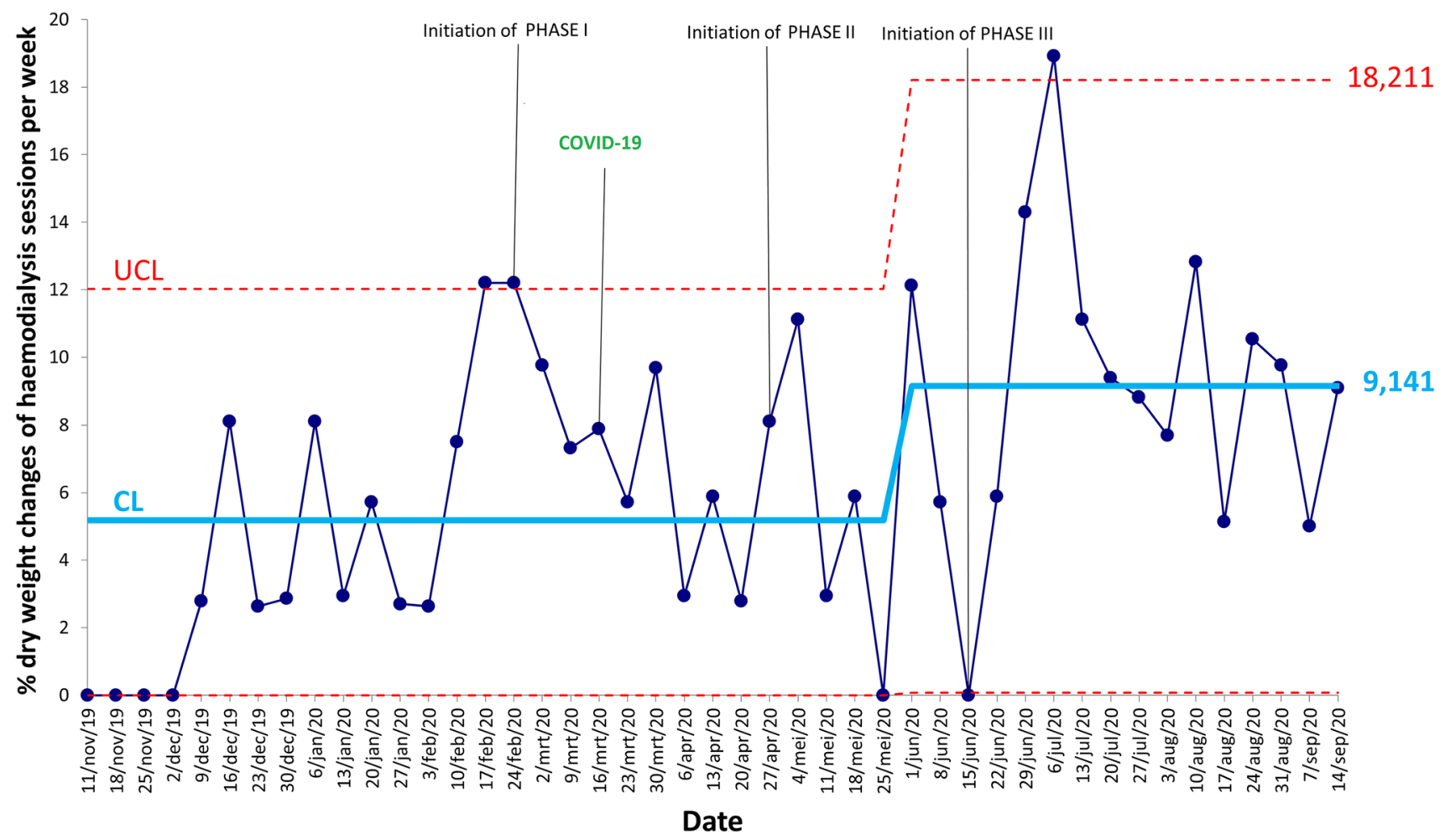

Fig. 4 Statistical control chart (c-chart) of process measure: dry weight changes. Red-dotted lines represent, respectively, upper control limit (UCL, $+3 \sigma$ to mean) and lower control limit (LCL, -3 $\sigma$ from mean). Light blue line represents control line (CL, mean). PHASE I: Multidisciplinary dry weight meetings with standard-

post-intervention compared to $13.3 \pm 6.6 \%$ pre-intervention. The sustained decrease in IDH following implementation of our intervention was not at the expense of more fluid overloaded patients, as the proportion of hypertensive patients remained similar and even a drop in antihypertensive agent use was noted from $22.1 \pm 1.4 \%$ at baseline to $14.8 \pm 0.9 \%$ post-intervention $(p<0.05)$. Similar results were obtained by Tai et al. [27] who performed a QI project in an adult haemodialysis unit demonstrating a successful decrease in IDH events by implementing an educational program on fluid volume management for physicians and nurses [27].

We believe that three aspects of the PedHDfluid program contributed to the success of the program: (i) multidisciplinary approach, (ii) educational content and (iii) use of electronic health records. First, approaching dry weight assessment with a multidisciplinary team has been recommended by several authors $[3,28]$. The integration of the information provided by a dietician and nurses in combination with fluid volume parameters (such as blood pressure, intra-dialytic weight gain and blood chemistry) is key to obtain individualized and patient-centred fluid volume assessment. Our dietician provided input during dry weight ized dry weight assessment. PHASE II: Automatically generated dry weight flow sheet. PHASE III: An introductory presentation for new fellows and dialysis nurses. COVID-19: presents time point of changes in the dialysis unit due to the COVID-19 pandemic, identified as a contextual factor that may have interfered with our project

meetings on the patient's dietary salt intake, intra-dialytic weight gain and nutritional status, while our dialysis nurses provided input on fluid removal tolerability, dialysis events and patient symptomatology. A second contributor to the success of the program was our focus on education. During the weekly dry weight meetings, there was the opportunity for staff physicians to incorporate teaching and the trainees learned the nuanced assessment of dry weight from these rich discussions between physicians, nurses and dietitians that incorporated the clinical data available including blood pressure, weights, blood volume monitoring and bioimpedance studies. This is in line with the significant decrease in IDH events found by Tai et al. [27] after implementing an educational program that incorporated IDH pathophysiology, critical reviews of studies on IDH strategies and small group discussions on difficult cases in their unit [27].

The third contributor to the success of this QI project is the ease with which longitudinal parameters related to dry weight assessment could be tracked in the Epic ${ }^{\circledR}$ "Dry Weight Evaluation" flow sheet and "Dry Weight Assessment" Synopsis. Dry weight is somewhat of a moving target that needs close monitoring and regular adjustment, 
especially in growing children, and this is best achieved by incorporating a number of relevant parameters and reviewing trends over time rather than depending on one parameter at a random time point [27]. By automatically collating all relevant parameters over a period of time in one place, the Epic ${ }^{\circledR}$ "Dry Weight Evaluation" flow sheet and "Dry Weight Assessment" Synopsis facilitated the assessment of dry weight at the patient's bedside. Previous studies targeting enhanced multifaceted dry weight estimation by using artificial intelligence tools have been able to demonstrate similar results. For example, Niel et al. [29] developed an artificial intelligence tool to assist dry weight estimation in the paediatric dialysis unit using patients' hydration status, relative blood volume and blood pressure, in which they demonstrated that in most cases, the dry weight-assisted tool outperformed in predicting dry weight in comparison to experienced nephrologists [29].

While the PedHDfluid decreased hypotension, UF cessation and IDH events in our study, no change in fluid bolus administration was seen. The reason why we could not decrease fluid bolus administration is unclear but might be due to an increased awareness on IDH by the program, resulting in more easy administration of fluid boluses. Moreover, the pathophysiology of IDH is more complex than solely poor fluid volume management. Poor compensatory mechanisms such as abnormal cardiac function and autonomic failure are known contributors to IDH, but these were not addressed by our PedHDfluid program.

A particular strength of this study is the development of a novel dry weight flow sheet incorporated into a widely used electronic health record that can be used by other paediatric dialysis units. Developing this novel assessment and tracking tool will allow other centres to incorporate this tool into their own practice, enabling dry weight assessment and fluid volume management at the bedside in these other paediatric dialysis centres.

Our study has some limitations. First, one contextual factor that may have interfered with our project was the COVID-19 pandemic (especially the lockdown period between March and May 2020). Our group pivoted to running our dry weight meetings on a virtual platform and clinical fellows were divided into two groups: one that worked on-site in the dialysis unit and one that participated in virtual clinics off-site. This change made fellows rotate more frequently (every 2 weeks as opposed to every 2 months) between different clinical services, a system that naturally affected the longitudinal aspect of care provided by the dialysis fellow which may have impacted our results. Moreover, HD schedules were altered to limit the number of hospital visits for each patient and the theoretical exposure risk for dialysis personnel (for example converting from a 4 times per week 3 -h dialysis schedule to a 3 times per week 4 -h schedule). This change in scheduling may have interfered with our project in different directions. While a decrease in dialysis frequency with subsequently greater ultrafiltration targets contributed to a greater likelihood of IDH events, the longer dialysis sessions could allow more time to remove fluid and protected our patients from IDH. However, no sustained changes in dialysis frequency nor dialysis duration were noted between the baseline registration and the post-intervention phase in our study. A second limitation of this study is the absence of detailed symptom registration. While symptoms are a critical consideration in the definition of IDH, our project did not collect data on symptoms present in our patients as symptoms are nonspecific, difficult to register in a non-verbal paediatric population and no validated tool is available. However, our teaching program and dry weight meetings specifically addressed symptoms to standardize practices among nursing staff. Finally, as the PedHDfluid program was only implemented and assessed in a single centre, future studies are needed to validate and confirm these findings in other dialysis units.

\section{Conclusion}

This study demonstrates that changing the culture and policy in a dialysis unit on fluid volume management can significantly and sustainably decrease IDH events, and subsequently improve patient outcomes while on HD. Since clear international consensus guidelines on paediatric HD fluid volume management are lacking, this initiative might inform other paediatric dialysis units about IDH event reduction strategies and training, as well as offer a tool embedded in the Epic $\AA$ electronic health record that can be used in other centres.

Supplementary Information The online version contains supplementary material available at https://doi.org/10.1007/s00467-021-05298-z.

Acknowledgements Paediatric nephrology trainees participate in the Paediatric Co-Learning Curriculum in Quality Improvement (CCQI) (https://www.deptmedicine.utoronto.ca/co-learning-curri culum-quality-improvement). This initiative is led by the Centre for Quality Improvement \& Patient Safety (C-QuIPS) at the University of Toronto. Here, they participate in a formal longitudinal QI curriculum from September to June alongside other Divisions in the Department of Paediatrics. The curriculum consists of interactive learning workshops, a team-based QI project with faculty mentors, QI coaching sessions and a final project presentation at the University of Toronto, QI Day. The authors thank all the dialysis nurses and our dietician Andrea Aquilina for their willingness to try new approaches and to their daily commitment to create an outstanding culture of dialysis care. We also thank the Paediatric Nephrology fellows (2019-2020) Motasem Doudin, Donna Austin, Priya Saini, Magdalena Riedl and Lindo Mahlase for their contributions, hard work and input in this project. At last, a special word of thanks to the Epic ${ }^{\circledR}$ analysts Lindsay Viau and Amanda Blackwell for building a practical and efficient dry weight flow sheet in Epic ${ }^{\circledR}$. 
Author contributions Conceptualization: SE, WS, JNA, BV, MA, KA, NR, TCW, LC, ND, HE and ZM. Methodology: SE, WS, JNA, BV, KA, NR, LC and ND. Formal analysis and investigation: SE, WS, JNA and ND. Writing - original draft preparation: SE, WS and JNA. Writingreview and editing: SE, WS, JNA, BV, MA, KA, NR, TCW, LC, ND HE and ZM. Resources: HE, ZM, ND, TCW, LC and NR. Supervision: HE, ZM, ND, TCW, LC and NR.

\section{Funding None.}

Data availability The datasets generated during and/or analysed during the current study are available from the corresponding author on reasonable request.

Code availability Not applicable.

\section{Declarations}

Conflict of interest The authors declare that they have no conflict of interest.

Ethical approval This study was reviewed and approved through The Hospital for Sick Children's Quality Improvement Review Process (Toronto, Canada), ID: QIP-2020-02-05. The requirement for consent was waived.

Consent to participate Not applicable.

Consent for publication Not applicable.

\section{References}

1. Raina R, Lam S, Raheja H, Krishnappa V, Hothi D, Davenport A et al (2019) Pediatric intradialytic hypotension: recommendations from the pediatric continuous renal replacement therapy (PCRRT) workgroup. Pediatr Nephrol 34:925-941. https://doi.org/10.1007/ s00467-018-4190-1

2. Kuipers J, Verboom LM, Ipema KJR, Paans W, Krijnen WP, Gaillard C et al (2019) The prevalence of intradialytic hypotension in patients on conventional hemodialysis: a systematic review with meta-analysis. Am J Nephrol 49:497-506. https://doi.org/10.1159/ 000500877

3. Reeves PB, Mc Causland FR (2018) Mechanisms, clinical implications, and treatment of intradialytic hypotension. Clin J Am Soc Nephrol 13:1297-1303. https://doi.org/10.2215/CJN.12141017

4. Hayes W, Hothi DK (2011) Intradialytic hypotension. Pediatr Nephrol 2:867-879. https://doi.org/10.1007/s00467-010-1661-4

5. Hothi DK (2009) An investigation into the mechanisms, consequences and moderators of intradialytic hypotension in pediatric hemodialysis. Thesis submitted to University College London for the degree of Doctor of Medicine (Research). Institute of Child Health \& Great Ormond Street Hospital for Children. https://discovery.ucl.ac.uk/id/eprint/17442/1/17442. pdf. Accessed Aug 1, 2021

6. Kooman J, Basci A, Pizzarelli F, Canaud B, Haage P, Fouque $D$ et al (2007) EBPG guideline on haemodynamic instability. Nephrol Dial Transplant 22(Suppl 2):ii22-ii44. https://doi.org/ 10.1093/ndt/gfm019

7. Converse RL Jr, Jacobsen TN, Jost CM, Toto RD, Grayburn PA, Obregon TM et al (1992) Paradoxical withdrawal of reflex vasoconstriction as a cause of hemodialysis-induced hypotension. J Clin Invest 90:1657-1665. https://doi.org/10.1172/JCI116037
8. Stefansson BV, Brunelli SM, Cabrera C, Rosenbaum D, Anum E, Ramakrishnan $\mathrm{K}$ et al (2014) Intradialytic hypotension and risk of cardiovascular disease. Clin J Am Soc Nephrol 9:2124-2132. https://doi.org/10.2215/CJN.02680314

9. Shoji T, Tsubakihara Y, Fujii M, Imai E (2004) Hemodialysisassociated hypotension as an independent risk factor for two-year mortality in hemodialysis patients. Kidney Int 66:1212-1220. https://doi.org/10.1111/j.1523-1755.2004.00812.x

10. Flythe JE, Inrig JK, Shafi T, Chang TI, Cape K, Dinesh K et al (2013) Association of intradialytic blood pressure variability with increased all-cause and cardiovascular mortality in patients treated with long-term hemodialysis. Am J Kidney Dis 61:966-974. https://doi.org/10.1053/j.ajkd.2012.12.023

11. McIntyre CW, Burton JO, Selby NM, Leccisotti L, Korsheed S, Baker CS et al (2008) Hemodialysis-induced cardiac dysfunction is associated with an acute reduction in global and segmental myocardial blood flow. Clin J Am Soc Nephrol 3:19-26. https:// doi.org/10.2215/CJN.03170707

12. Flythe JE, Xue H, Lynch KE, Curhan GC, Brunelli SM (2015) Association of mortality risk with various definitions of intradialytic hypotension. J Am Soc Nephrol 26:724-734. https://doi.org/ 10.1681/ASN.2014020222

13. Chang TI, Paik J, Greene T, Desai M, Bech F, Cheung AK et al (2011) Intradialytic hypotension and vascular access thrombosis. J Am Soc Nephrol 22:1526-1533. https://doi.org/10.1681/ASN. 2010101119

14. Jansen MA, Hart AA, Korevaar JC, Dekker FW, Boeschoten EW, Krediet RT et al (2002) Predictors of the rate of decline of residual renal function in incident dialysis patients. Kidney Int 62:10461053. https://doi.org/10.1046/j.1523-1755.2002.00505.x

15. MacEwen C, Sutherland S, Daly J, Pugh C, Tarassenko L (2017) Relationship between hypotension and cerebral ischemia during hemodialysis. J Am Soc Nephrol 28:2511-2520. https://doi.org/ 10.1681/ASN.2016060704

16. Burton JO, Jefferies HJ, Selby NM, McIntyre CW (2009) Hemodialysis-induced cardiac injury: determinants and associated outcomes. Clin J Am Soc Nephrol 4:914-920. https://doi.org/10. 2215/CJN.03900808

17. Dasgupta I, Thomas GN, Clarke J, Sitch A, Martin J, Bieber B et al (2019) Associations between hemodialysis facility practices to manage fluid volume and intradialytic hypotension and patient outcomes. Clin J Am Soc Nephrol 14:385-393. https://doi.org/10. 2215/cjn.08240718

18. Mitchell S (2002) Estimated dry weight (EDW): aiming for accuracy. Nephrol Nurs J 29:421-428 (quiz 429-430)

19. Flynn JT, Kaelber DC, Baker-Smith CM, Blowey D, Carroll AE, Daniels SR, de Ferranti SD, Dionne JM, Falkner B, Flinn SK, Gidding SS, Goodwin C, Leu MG, Powers ME, Rea C, Samuels J, Simasek M, Thaker VV, Urbina EM, Subcommittee on screening and management of high blood pressure in children (2017) Clinical practice guideline for screening and management of high blood pressure in children and adolescents. Pediatrics 140:e20171904. https://doi.org/10.1542/peds.2017-1904

20. McQuillan RF, Silver SA, Harel Z, Weizman A, Thomas A, Bell $\mathrm{C}$ et al (2016) How to measure and interpret quality improvement data. Clin J Am Soc Nephrol 11:908-914. https://doi.org/10.2215/ CJN.11511015

21. Goodman D, Ogrinc G, Davies L, Baker GR, Barnsteiner J, Foster TC et al (2016) Explanation and elaboration of the SQUIRE (Standards for Quality Improvement Reporting Excellence) guidelines, V.2.0: examples of SQUIRE elements in the healthcare improvement literature. BMJ Qual Saf 25:e7. https://doi.org/10. 1136/bmjqs-2015-004480

22. Ogrinc G, Davies L, Goodman D, Batalden P, Davidoff F, Stevens D (2015) Squire 2.0 (Standards for Quality Improvement Reporting Excellence): revised publication guidelines from a detailed 
consensus process. Am J Crit Care 24:466-473. https://doi.org/ 10.4037/ajcc2015455

23. Weiner DE, Brunelli SM, Hunt A, Schiller B, Glassock R, Maddux FW et al (2014) Improving clinical outcomes among hemodialysis patients: a proposal for a "volume first" approach from the chief medical officers of US dialysis providers. Am J Kidney Dis 64:685-695. https://doi.org/10.1053/j.ajkd.2014.07.003

24. Assimon MM, Flythe JE (2020) Ultrafiltration rate and residual kidney function decline: yet another good reason to ask about urine. Am J Kidney Dis 75:322-324. https://doi.org/10.1053/j. ajkd.2019.10.007

25. Assimon MM, Wang L, Flythe JE (2018) Failed target weight achievement associates with short-term hospital encounters among individuals receiving maintenance hemodialysis. J Am Soc Nephrol 29:2178-2188. https://doi.org/10.1681/ASN.2018010004

26. Arneson TJ, Liu J, Qiu Y, Gilbertson DT, Foley RN, Collins AJ (2010) Hospital treatment for fluid overload in the medicare hemodialysis population. Clin J Am Soc Nephrol 5:1054-1063. https://doi.org/10.2215/CJN.00340110

27. Tai DJ, Conley J, Ravani P, Hemmelgarn BR, MacRae JM (2013) Hemodialysis prescription education decreases intradialytic hypotension. J Nephrol 26:315-322. https://doi.org/10.5301/jn.5000147

28. Chazot $C$ (2009) Can chronic volume overload be recognized and prevented in hemodialysis patients? Use of a restricted-salt diet. Semin Dial 22:482-486. https://doi.org/10.1111/j.1525-139X. 2009.00642.x

29. Niel O, Bastard P, Boussard C, Hogan J, Kwon T, Deschênes G (2018) Artificial intelligence outperforms experienced nephrologists to assess dry weight in pediatric patients on chronic hemodialysis. Pediatr Nephrol 33:1799-1803. https://doi.org/10.1007/ s00467-018-4015-2

Publisher's note Springer Nature remains neutral with regard to jurisdictional claims in published maps and institutional affiliations. 